\title{
Two-Dimensional Hexagonal Boron Nitride Nanosheets as Lateral Heat Spreader With High Thermal Conductivity
}

OPEN ACCESS

Edited by:

Xiaoliang Zeng,

Shenzhen Institutes of Advanced Technology (CAS), China

Reviewed by:

Xuebin Wang,

Nanjing University, China

Peng Ding,

Shanghai University, China

Kang Zheng,

Institute of Solid State Physics, Hefel Institutes of Physical Science (CAS),

China

Chuntai Liu,

Zhengzhou University, China

${ }^{*}$ Correspondence:

Zhongbin Pan

panzhongbin@163.com

Xinfeng Wu

xfwu@shmtu.edu.cn

Jinhong Yu

yujinhong@nimte.ac.cn

${ }^{t}$ These authors have contributed

equally to this work

Specialty section:

This article was submitted to Polymeric and Composite Materials,

a section of the journal

Frontiers in Materials

Received: 17 November 2021 Accepted: 31 December 2021

Published: 21 January 2022

Citation:

Kong X, Li L, Li M, Xia J, Wang Y, Wei X, Xiong S, Gong P, Pan Z, Wu X, Cai $T$, Nishimura $K$, Lin $C-T$, Jiang $N$

and Yu J (2022) Two-Dimensional Hexagonal Boron Nitride Nanosheets as Lateral Heat Spreader With High

Thermal Conductivity.

Front. Mater. 8:817061.

doi: 10.3389/fmats.2021.817061
Xiangdong Kong ${ }^{1,2 t}$, Linhong $\mathrm{Li}^{2,3 t}$, Maohua $\mathrm{Li}^{2}$, Juncheng $\mathrm{Xia}^{2}$, Yandong Wang ${ }^{2}$, Xianzhe $\mathrm{Wei}^{2}$, Shaoyang Xiong ${ }^{2}$, Ping Gong ${ }^{2}$, Zhongbin Pan ${ }^{1 \star}$, Xinfeng $\mathrm{Wu}^{4 \star}, \mathrm{Tao} \mathrm{Cai}^{2}$, Kazuhito Nishimura ${ }^{5}$, Cheng-Te Lin ${ }^{2,3}$, Nan Jiang ${ }^{2,3}$ and Jinhong $\mathrm{Yu}^{2,3 *}$

${ }^{1}$ School of Materials Science and Chemical Engineering, Ningbo University, Ningbo, China, ${ }^{2}$ Key Laboratory of Marine Materials and Related Technologies, Zhejiang Key Laboratory of Marine Materials and Protective Technologies, Ningbo Institute of Materials Technology and Engineering, Chinese Academy of Sciences, Ningbo, China, ${ }^{3}$ Center of Materials Science and Optoelectronics Engineering, University of Chinese Academy of Sciences, Beijing, China, ${ }^{4}$ College of Ocean Science and Engineering, Shanghai Maritime University, Shanghai, China, ${ }^{5}$ Advanced Nano-processing Engineering Lab, Mechanical Engineering, Kogakuin

University, Tokyo, Japan

A highly thermally conductive heat spreader for applications in electronic devices is becoming increasingly demanding, and therefore the removal of excess heat requires an efficient heat dissipating device. Boron nitride nanosheets (BNNSs) were prepared as thermally conductive fillers using hexagonal boron nitride ( $h-B N$ ) powder as raw material by a water exfoliation method. A composite film was prepared by vacuum filtration using cellulose nanofibers (CNFs) as the substrate with an in-plane thermal conductivity (TC) of 82.4 $\mathrm{W} \mathrm{m}^{-1} \mathrm{~K}^{-1}$, thermal conductivity enhancement increasing by $9,486 \%$ compared to pure cellulose film. Thus, CNF/BNNS composite films are promising as effective thermal interface materials (TIMs) in electronic devices and electronic component applications.

Keywords: boron nitride nanosheets, cellulose nanofibers, heat spreader, filtration, thermal conduction

\section{INTRODUCTION}

In recent years, with the continuous improvement of technology, high-power electronic devices and highly integrated electronic components are also developed towards more precision and miniaturization. However, a lot of problems, such as waste heat, have also arisen. When the heat generated by a device is not effectively removed during prolonged periods of high workload, the device's efficiency, service life, and reliability suffer significantly. The air gap between the heat sink and the heat-generating device has a very low thermal conductivity, resulting in a relatively high contact thermal resistance. Thermal interface materials can bridge this air gap, lowering contact thermal resistance and improving thermal transportation performance. The development of heat spreader with efficient heat dissipation properties to remove excess heat has therefore become a hot topic of research (Cui et al., 2020; Yan et al., 2021a). Efficient heat dissipation will be a critical factor in developing devices and electronic components.

Anisotropic insulating 2D materials have a high thermal conductivity along the planar aspect compared to isotropic conductive materials, effectively blocking the influence between components (Yang et al., 2021b). The two-dimensional (2D) BNNS has been widely used as thermal conductivity due to its high thermal conductivity (Yan et al., 2021b), excellent electrical insulation, and low dielectric constant (Wang et al., 2018; Hou et al., 2021). The thermal conductivity of BNNS has been 
reported to be in the range of $1700-2000 \mathrm{~W} \mathrm{~m}^{-1} \mathrm{~K}^{-1}$ (Zeng et al., 2011; Zeng et al., 2012). However, the high brittleness and low tensile strength of pure BNNS ( 17 MPa) (Wu et al., 2017) limit its application. For the electronic miniaturization components, a thermal interface material with better mechanical properties and thermal conductivity is needed. Filling BNNS with polymers that serve as adhesive can build up a thermally conductive network, which is a reliable way to reduce the brittleness and increase the tensile strength of the composite.

Cellulose nanofibres (CNF) are low-cost, all-natural, inexhaustible polymeric materials prepared from the cellulose of plant origin. Due to its excellent mechanical and thermal properties (Zhou et al., 2016), cellulose is increasingly being used as a substrate for thin film research (Wu et al., 2017; Wu et al., 2018). It has previously been reported that two-dimensional BNNS can be dispersed in aqueous suspension by electrostatic repulsion and spatial site resistance (Zhu et al., 2014; Li et al., 2015).

In this paper, we demonstrate a simple water exfoliation method to obtain BNNS (Wang et al., 2018) and introduce BNNS into a cellulose substrate to prepare a composite film by vacuum filtration. Firstly, we prepared CNF/BNNS composite films by controlling the suspension concentration at $0.15 \%$ and increasing the BNNS filler content. We found that the thermal conductivity of the CNF/BNNS composite films increased with increasing BNNS filler content. When the BNNS filler concentration was $90 \%$, the CNF/BNNS composite films had the highest thermal conductivity of $70.4 \mathrm{~W} \mathrm{~m}^{-1} \mathrm{~K}^{-1}$. Secondly, to further improve the thermal conductivity of the CNF/BNNS composite films, we consider making the BNNS in the CNF/ BNNS composite films have a more regular horizontal arrangement to form a better heat conduction path. We decided to control the same BNNS filler content at $90 \%$ and change the different suspension concentration for comparison. It was found that the suspension concentration had a significant effect on the CNF/BNNS composite films, with a significantly higher thermal conductivity of $82.4 \mathrm{~W} \mathrm{~m}^{-1} \mathrm{~K}^{-1}$ at a suspension concentration of $0.1 \%$. We have investigated the in-plane thermal conductivity of the CNF/BNNS composite films in these two ways, particularly the effect of varying the suspension concentration with a $90 \%$ BNNS filler content. The composite films show good thermal conductivity and can be used as a heat spreader for electronic devices (Yang et al., 2021a; Zhang et al., 2021).

\section{EXPERIMENTAL}

\section{Materials}

Hexagonal boron nitride (lateral size $1-5 \mu \mathrm{m}$ ) powder from ESK Ceramics (Germany). Cellulose raw material obtained from Guilin Qihong Technology Co. (Product model CNF-P, Content of $1.1 \mathrm{wt} \%$, The raw material is wood pulp, diameter

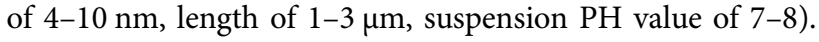

\section{Preparation of BNNS}

The h-BN powder $(80 \mathrm{~g})$ and $4,000 \mathrm{ml}$ of deionized water were dispersed. This dispersion was subjected to the first step of the stripping process with a cutting-edge ultrasound machine, which ultrasonicated the h-BN micronized powder for $120 \mathrm{~min}$. After sonication, stand for $5 \mathrm{~h}$ and separate into layers, then pour out the upper dispersion and dry to obtain BNNS. And it should be noted at this point that the $80 \mathrm{~g}$ of h-BN powder was not completely exfoliated. The solvent of the sediment below could be reused as the original solvent in another liquid stripping process. It means that the residue can be re-dispersed in $4,000 \mathrm{ml}$ of deionized water, sonicated, filtered. The above procedure is repeated after several cycles to achieve complete exfoliation.

\section{Preparation of Composite Films}

Herein, the vacuum filtration method was used to prepare $\mathrm{CNF} /$ BNNS composite films. We used CNF/BNNS composite films with a suspension concentration of $0.1 \mathrm{wt} \%$ and $90 \mathrm{wt} \%$ BNNS content as an example. In the first step, $0.09 \mathrm{~g}$ BNNS and $1.0 \mathrm{~g}$ cellulose slurry were dispersed in $99 \mathrm{ml}$ of deionized water in a mixing cylinder with a speed mixer (Shanghai Acclaim International Trading Co. Model: DAC 150.1 FVZ-K) at $3,500 \mathrm{rpm}$, where the deionized water was added twice. The purpose of this is to prevent BNNS from sticking to the wall of the mixing cylinder and causing uneven dispersion of BNNS. In the second step, the prepared dispersion was further dispersed in the sonicator for another $30 \mathrm{~min}$ to obtain the final suspension by adding $33 \mathrm{ml}$ and vacuum filtering through a polycarbonate membrane with a pore size of $0.22 \mu \mathrm{m}$ to obtain a CNF/BNNS composite film with a thickness of about $30 \mu \mathrm{m}$. The preparation procedure of CNF/BNNS composite film was shown in Figure 1.

\section{Characterization}

Both BNNS and composite films were observed with a Regulus 8230 scanning electron microscope (HITACHI, Regulus 8230, Japan). In order to prevent charge accumulation on the cross section of the composite film, we pre-sprayed a layer of platinum on the surface of the composite film. Transmission electron microscope (Thermo Fisher Scientific, Talos, MA, United States). Atomic force microscope (AFM, SPM Dimension 3100, Veeco, NY, United States). Raman spectroscopy using a laser confocal micro Raman spectrometer (HORIBA FRANCE SAS, LabRAM Odyssey, Japan) with a laser wavelength of $532 \mathrm{~nm}$. The crystalline structure and surface chemical compositions were determined using X-ray diffractometer (XRD, D8 Discover/GADDS, Bruker, Germany) with $\mathrm{Cu}$ Karadiation. Infrared spectroscopy was performed using a FTIR spectrometer (Thermo, Nicolet is 50, MA, United States). The thermal conductivity of the composite film was determined using the LFA 467 Nanoflash (NETZSCH, Germany). Infrared photos were taken by an infrared camera (Fluke, Ti400, WA, United States).

\section{RESULTS AND DISCUSSION}

\section{Characterizations of BNNS}

As shown in Figure 2A the size distribution of BNNS is from 1 to $5 \mu \mathrm{m}$ with a flat and regular surface, proving the BNNS was 

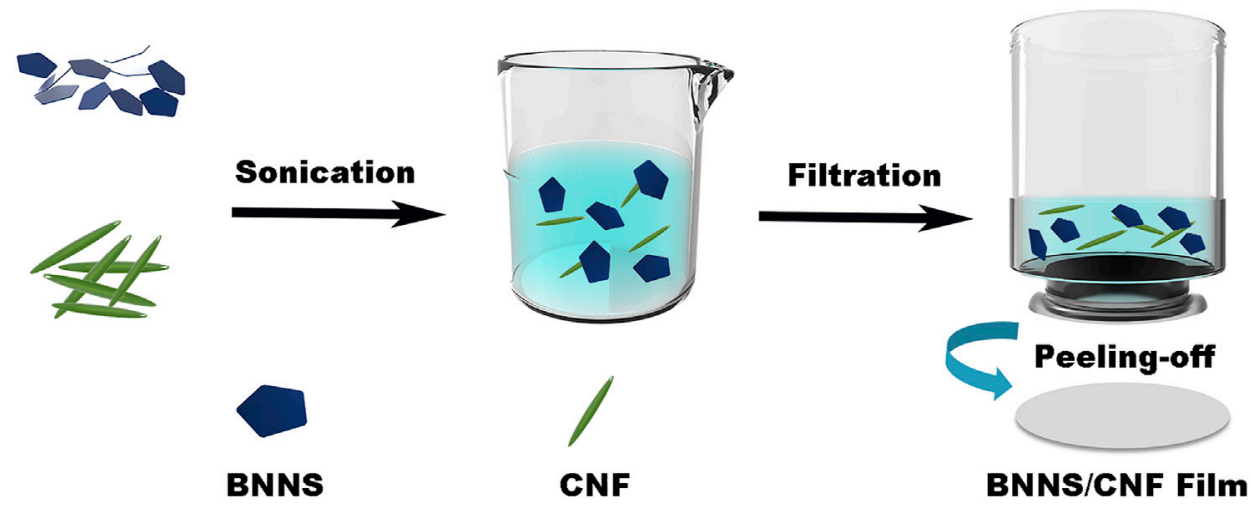

FIGURE 1 | Schematic diagram of the preparation of CNF/BNNS composite film.
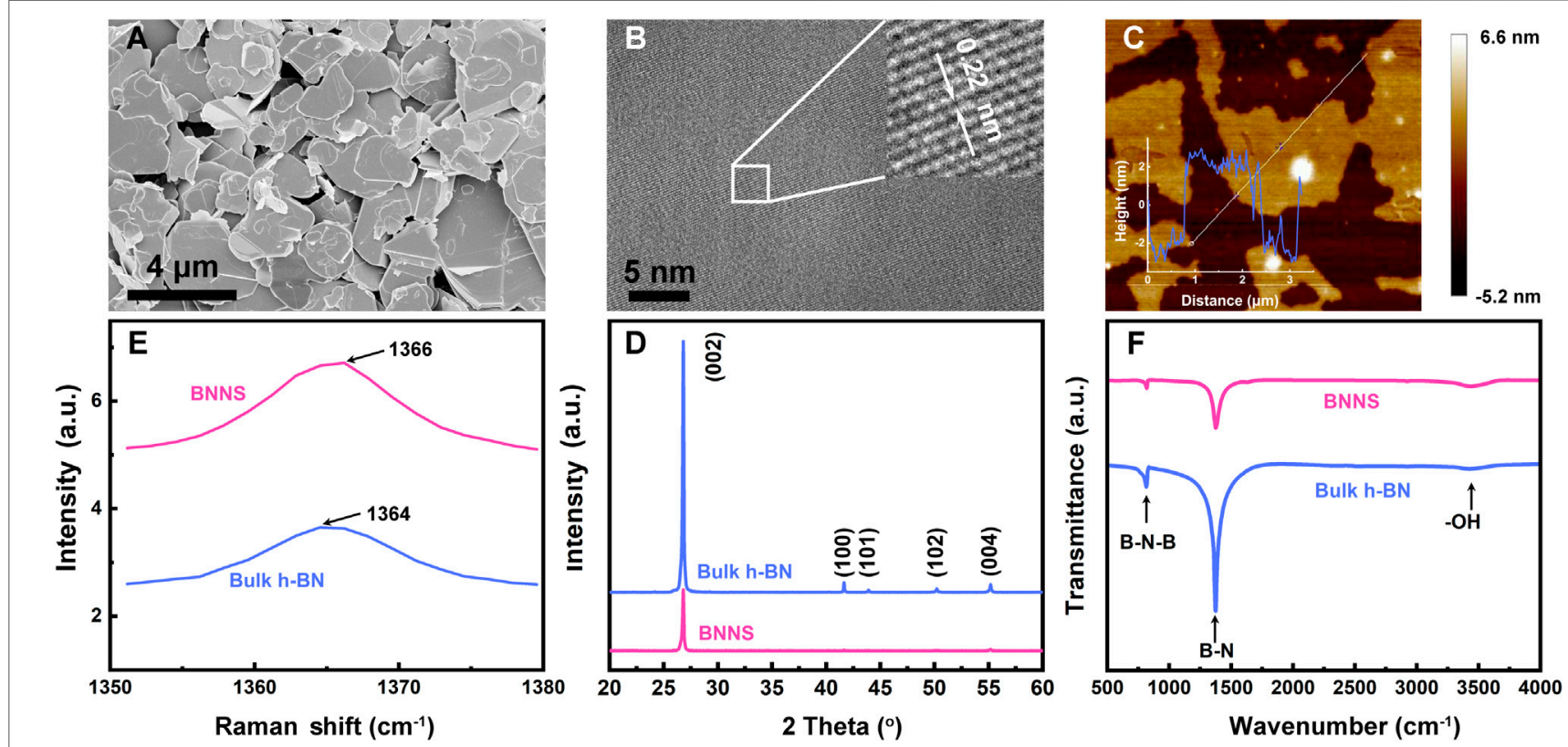

FIGURE 2 | Characterization of BNNS and BN powders: (A) SEM, (B) TEM, (C) AFM, (D) Raman, (E) XRD, and (F) FTIR spectra.

successfully exfoliated from h-BN. A clear lattice can be seen in the HRTEM image in Figure 2B, indicating that the exfoliated BNNS still maintains an ordered structure. The top right inset of Figure 2B shows a magnified view of the crystal lattice, which presents the crystal lattice spacing of $0.22 \mathrm{~nm}$. The thickness of BNNS was measured by an atomic force microscope (AFM). The scanned profile is shown in Figure 2C with a thickness of $3.1 \mathrm{~nm}$ for BNNS (Li et al., 2016; Zhu et al., 2016). The crystal structure and surface functional groups of BNNS have been studied to provide more information on the exfoliation of BNNS. The characteristics of BNNS by Raman spectral frequencies in the G-band of the original h-BN and the stripped BNNS are shown in Figure 2D. Compared to the original $\mathrm{h}-\mathrm{BN}\left(1,364 \mathrm{~cm}^{-1}\right)$, the BNNS $\left(1,366 \mathrm{~cm}^{-1}\right)$ is blue shifted and has an increased G-band frequency. As the number of boron nitride layers decreases, the in-plane strain in the boron nitride layer increases, and the interlayer interaction weakens, resulting in an increase in the G-band frequency. A good correspondence between h-BN and BNNS can be seen in the XRD diffraction peaks of Figure 2E, which are related to the (002), (100), (101), (102), and (004) surfaces, respectively, and it can be seen that h-BN is well exfoliated into BNNS. The FTIR spectra of $h-B N$ and BNNS in Figure 2F show a small peak at $816 \mathrm{~cm}^{-1}$ belonging to the $\mathrm{B}-\mathrm{N}$ stretching vibration $\left(\mathrm{E}_{1 \mathrm{u}}\right.$ mode) and a broad peak at $1,373 \mathrm{~cm}^{-1}$ belonging to the $\mathrm{B}-\mathrm{N}-\mathrm{B}$ out-of-plane bending deformation ( $\mathrm{A}_{2 \mathrm{u}}$ mode) (Shi et al., 2010; Lee et al., 2015). A peak at $3,440 \mathrm{~cm}^{-1}$ is due to $-\mathrm{OH}$ vibration(Pan et al., 2021). 

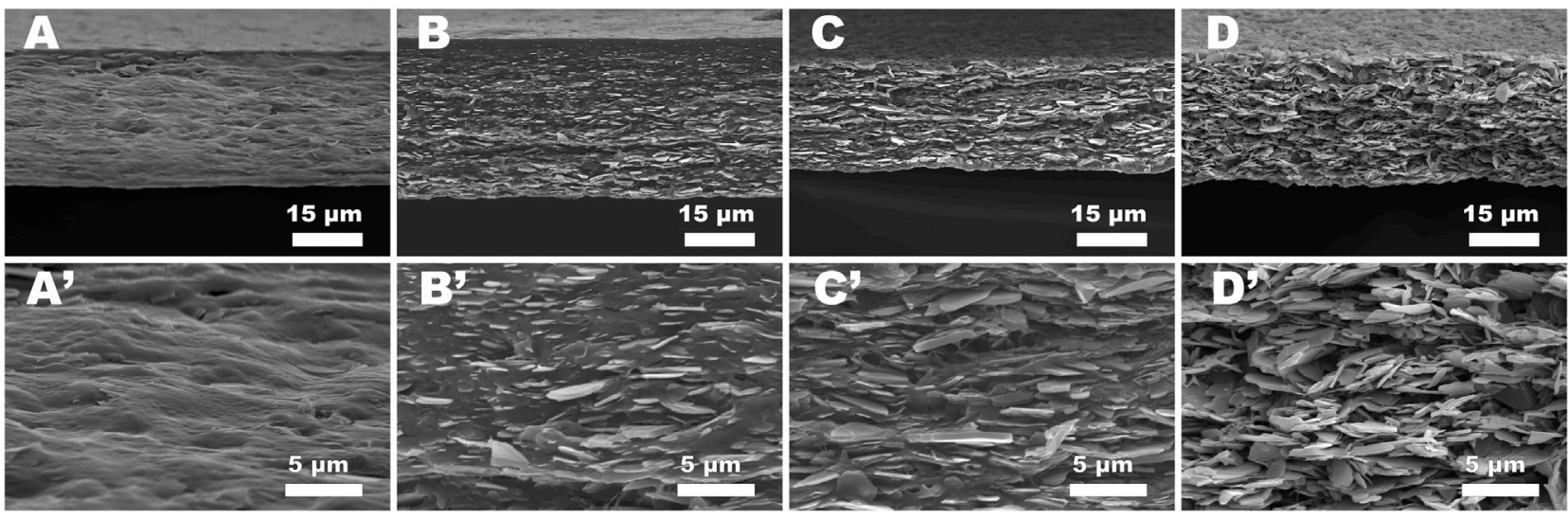

FIGURE 3 | Where (A'-D') is the (A-D) high magnification SEM image, which shows more clearly the BNNS arrangement in the composite films
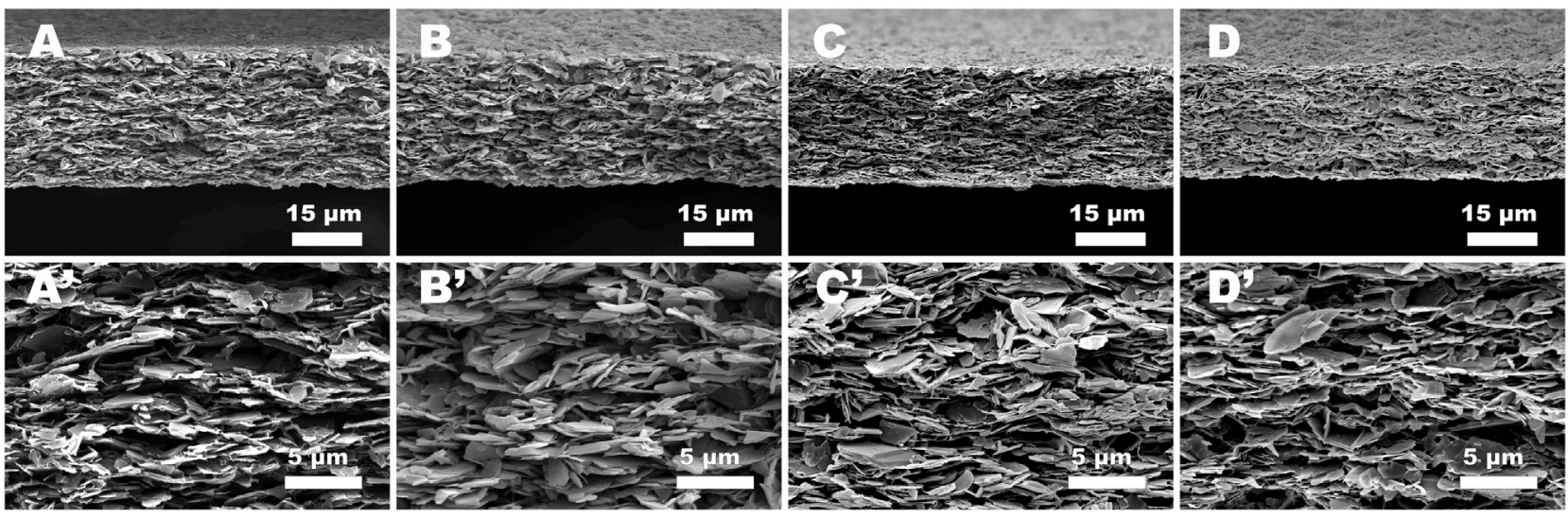

FIGURE 4 | Where (A'-D') is the (A-D) high magnification SEM image, which shows more clearly the BNNS arrangement in the composite films with different suspension concentrations.

\section{Microstructure of CNF/BNNS Composite Films}

The dispersion of the BNNS filler in the CNF matrix is an important influence on the properties of the composite ( $\mathrm{Wu}$ et al., 2017; Wu et al., 2018). The morphologies of the fractured surface of the composite films were investigated by scanning electron microscopy (SEM). Figures 3A,A', exhibit a CNF/BNNS composite film with a $10 \mathrm{wt} \%$ BNNS filler. Only a small amount of BNNS can be seen on the surface. With the increase of BNNS, more ordered BNNS appears on the fracture surface of the film, which provides more information for the improvement of the thermal conductivity of the composite film, as shown in Figures 3B-D. Figures 3B'-D' shows more details of Figures 3B-D. Firstly, we increased the BNNS filling amount by controlling the suspension concentration. Then change the suspension concentration under the same BNNS filling content, as shown in (Figures 4A,D') Figures 4A,A',B,B',C,C'D,D') are suspension concentrations of $0.1 \mathrm{wt} \%, 0.15 \mathrm{wt} \%, 0.3 \mathrm{wt} \%$ and $0.5 \mathrm{wt} \%$ respectively. Figures $\mathbf{4} \mathbf{A}^{\prime}-\mathbf{D}^{\prime}$ shows that as the suspension concentrations increase, the in-plane orientation of BNNS deteriorates, which is a key factor affecting the in-plane thermal conductivity of the CNF/BNNS composite film.

\section{Thermal Transportation Properties of CNF/ BNNS Composite Films}

We measured the thermal diffusion of the sample by laser flash at room temperature and pressure and then derived the thermal conductivity by the equation: $\lambda=\alpha \cdot \rho \cdot C_{p}$. Where $\lambda$ is the thermal conductivity, $\alpha$ is the thermal diffusion, $\rho$ is the density of the composite film, and $C P$ is the specific heat capacity(Yang et al., 2021a; Yang et al., 2021b). The results from Figure 5, show that controlling both the amount of BNNS filling and the concentration of the suspension results in enhanced thermal diffusion and thermal conductivity of the CNF/BNNS composite film in the in-plane direction (Zhou et al., 2016; Wu et al., 2017; Wu et al., 2018; Yan et al., 2021b). Figure $\mathbf{5 A}$ shows the variation of thermal diffusion and 

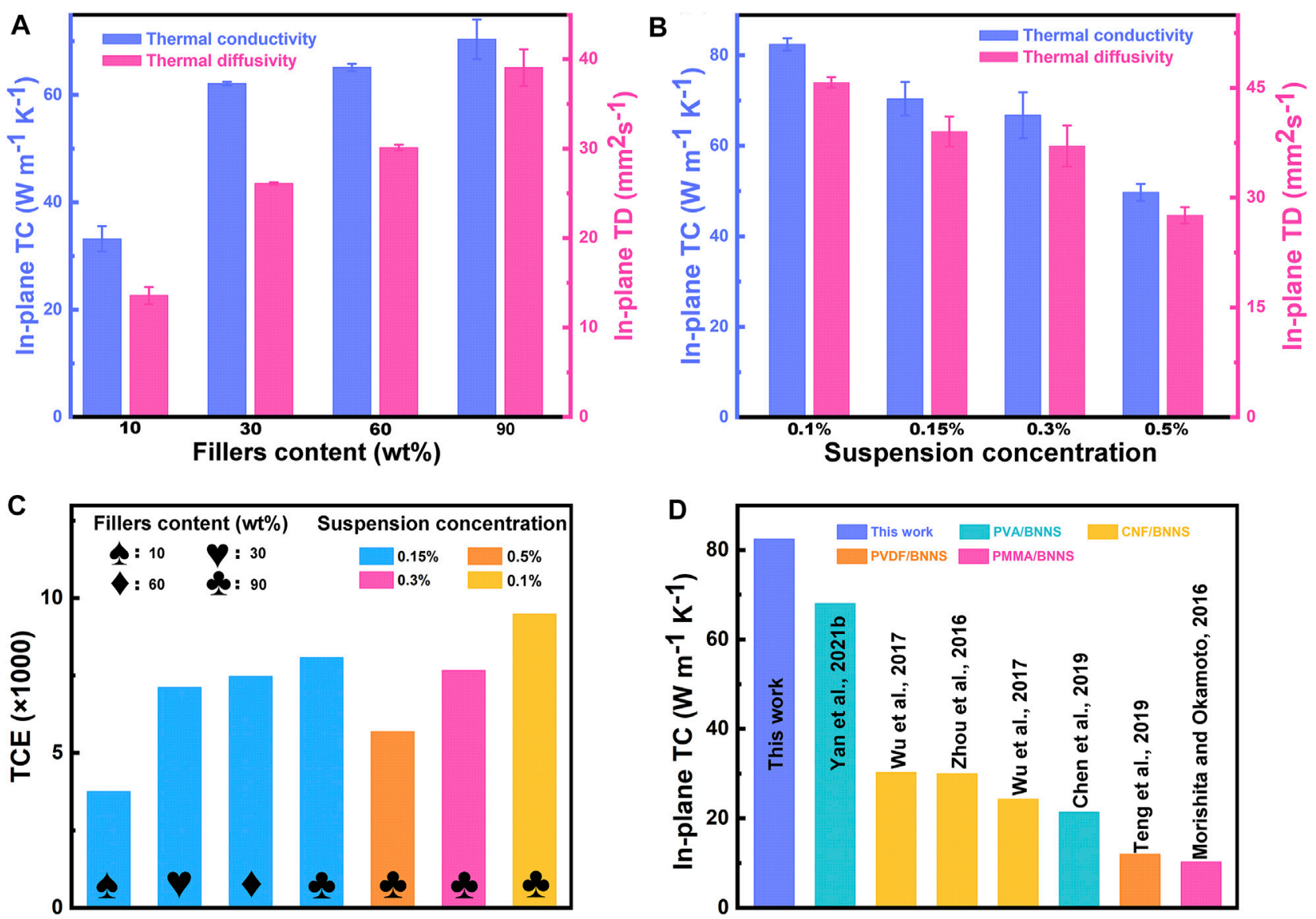

FIGURE 5 | Thermal properties of CNF/BNNS composite films: (A) Thermal conductivity (TC) and thermal diffusivity (TD) of CNF/BNNS composite films at different BNNS filler contents at a suspension concentration of 0.15 wt\%, (B) Thermal conductivity and thermal diffusivity of CNF/BNNS composite films with 90 wt\% BNNS filler content at different suspension concentrations, (C) Thermal conductivity enhancement (TCE), and (D) Comparison between our samples and BNNS/polymer composite films reported in the literature.

thermal conductivity with the increase of BNNS filler content when the suspension concentration is controlled to $0.15 \mathrm{wt} \%$. The thermal conductivity is $33.2 \mathrm{~W} \mathrm{~m}^{-1} \mathrm{~K}^{-1}$ at $10 \mathrm{wt} \%$ BNNS filler content and increases to $70.4 \mathrm{~W} \mathrm{~m}^{-1} \mathrm{~K}^{-1}$ at $90 \mathrm{wt} \%$ BNNS filler content (Yang et al., 2021b). The highly anisotropic thermal conduction of CNF/ BNNS composite films can be attributed to the particular stacking of BNNS layers in the CNF film. Furthermore, the boron nitride nanosheet is a two-dimensional thermal interface material with high thermal conductivity and low expansion coefficient. Its inplane thermal conductivity is much higher than the out-of-plane thermal conductivity. (Wu et al., 2017; Wang et al., 2018; Wu et al., 2018; Teng et al., 2019; Yan et al., 2021b; Yang et al., 2021b), So the in-plane thermal conductivity of the CNF/BNNS composite film increase as the filler content amount of BNNS in the network constructed by CNF continues to increase. When the BNNS filler content volume reaches $90 \%$, the thermal conductivity of the $\mathrm{CNF} /$ BNNS composite films is difficult to improve further. In order to improve the thermal conductivity of the CNF/BNNS composite films, we consider whether the thermal conductivity of the $\mathrm{CNF}$ / BNNS composite films is related to the concentration of the suspension. For this reason, we control the filler content amount of BNNS at $90 \%$ and change the suspension concentration to explore the effect on the thermal conductivity of the CNF/BNNS composite films. Figure 5B shows that when the BNNS filler content amount is $90 \mathrm{wt} \%$, the thermal diffusion, and thermal conductivity lower with the increase of suspension concentration. It can be seen from Figure 4 that the lower the concentration of the suspension, the better the arrangement of BNNS in the CNF/BNNS composite films, the more orderly heat conduction paths are formed, and the thermal conductivity continues to increase. The four samples' highest thermal conductivity test result was $82.4 \mathrm{~W} \mathrm{~m}^{-1} \mathrm{~K}^{-1}$ for the $0.1 \mathrm{wt} \%$ suspensions, and the lowest was $49.7 \mathrm{~W} \mathrm{~m}^{-1} \mathrm{~K}^{-1}$ for the $0.5 \mathrm{wt} \%$ suspensions(Yang et al., 2021b). Figure 5C shows the TCE used to compare our samples with the thermal conductivity enhancement of a pure cellulose matrix. TCE is expressed as TCE $=\frac{\lambda_{1}-\lambda_{0}}{\lambda_{0}} \times 100 \%$. Where $\lambda_{1}$ and $\lambda_{0}$ are the thermal conductivity of our sample and the pure cellulose matrix, respectively. The thermal conductivity enhancement increases by $9,486 \%$ compared to pure cellulose film. Figure 5D shows a comparison of the thermal conductivity of our work with that of other work (Morishita and Okamoto, 2016; Zhou et al., 2016; Wu et al., 2017; Wu et al., 2018; Chen et al., 2019; Teng et al., 2019). As a 


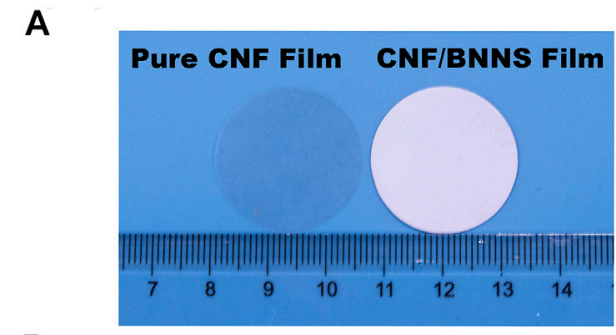

B

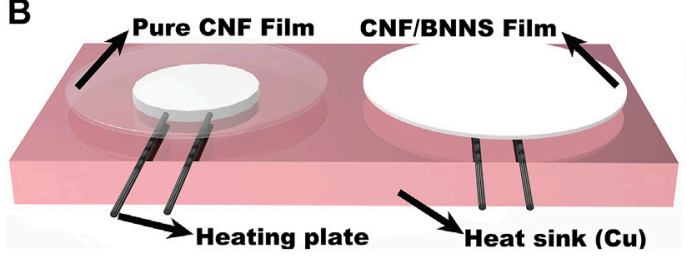

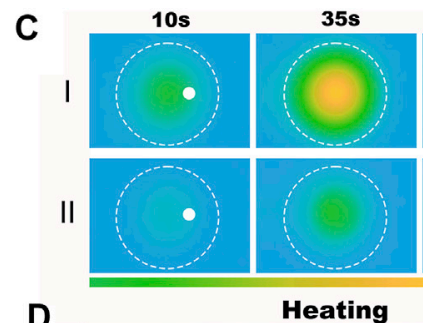

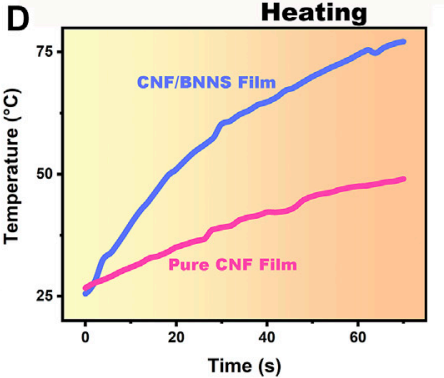

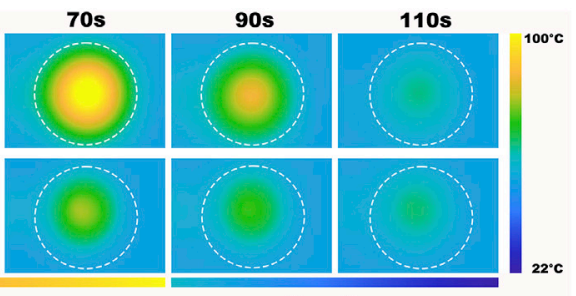

Cooling

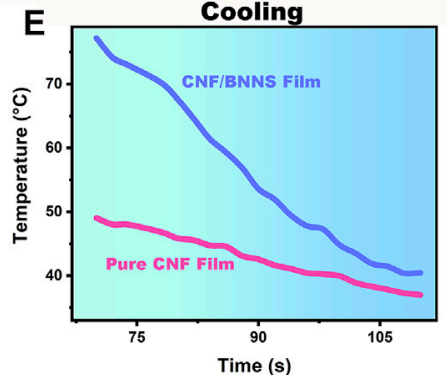

FIGURE 6 | (A) Photographs of pure CNF film and 90 wt\% BNNS content composite film, (B) Test model for IR images, (C) IR images of CNF/BNNS composite film with 90 wt\% BNNS content at a suspension concentration of 0.1 wt\% and pure CNF films heated and cooled with a ceramic heating plate heat source, (D) The heating curve and (E) the cooling curve as functions of time.

result, our CNF/BNNS composite film has higher thermal conductivity than other composites reported in previous studies.

Figure 6A shsows a pure cellulose film on the left and a CNF/ BNNS composite film with $90 \mathrm{wt} \%$ BNNS filler content at a suspension concentration of $0.1 \mathrm{wt} \%$ on the right. Figure 6B displayed a model of the system configuration when we took the IR images. Our sample, the ceramic heater, and the copper stage are from top to bottom. Figure $\mathbf{6 C}$ has been displayed IR images of CNF/BNNS composite films in I and pure cellulose films in II. The heating process is from 10 to 70 s, and the cooling process is from 70 to $110 \mathrm{~s}$. Figures 6D,E display the heating and cooling curve, respectively. The values of the heating and cooling curves come from the white marked points at the $10 \mathrm{~s}$ position in I and II, as shown in Figure 6C. It represents the process of a temperature change as the temperature spreads from the central point to the marked white point. It can be seen that the heating rate of our sample is much higher than that of pure cellulose film.

\section{CONCLUSION}

In summary, two-dimensional hexagonal boron nitride nanosheets as lateral heat spreaders were fabricated by a simple infiltrating method. Ultrahigh in-plane thermal

\section{REFERENCES}

Chen, J., Wei, H., Bao, H., Jiang, P., and Huang, X. (2019). MillefeuilleInspired Thermally Conductive Polymer Nanocomposites with Overlapping BN Nanosheets for Thermal Management Applications. ACS Appl. Mater. Inter. 11 (34), 31402-31410. doi:10.1021/ acsami.9b10810 conductivity of $82.4 \mathrm{~W} \mathrm{~m}^{-1} \mathrm{~K}^{-1}$ was obtained at $90 \mathrm{wt} \%$ BNNS filler content, which corresponds to a thermal conductivity enhancement increasing by $9,486 \%$ compared to pure CNF film. The high thermal conductivity of the composite film can be used as a heat spreader for electronic components and devices.

\section{DATA AVAILABILITY STATEMENT}

The raw data supporting the conclusion of this article will be made available by the authors, without undue reservation.

\section{AUTHOR CONTRIBUTIONS}

XK: Conceptualization, Methodology, Data Curation, Writing Original Draft. LL: Visualization, Investigation. ML: Investigation. JX: Investigation. YW: Resources. XW: Visualization. SX: Investigation. PG: Resources. ZP: Investigation, Resources XW: Resources. TC: Investigation. KN: Resources. TC: Validation. CL: Validation. NJ: Conceptualization, Supervision. JY: Conceptualization, Supervision, Writing-Review and Editing.

Cui, Y., Li, M., and Hu, Y. (2020). Emerging Interface Materials for Electronics thermal Management: Experiments, Modeling, and New Opportunities. J. Mater. Chem. C 8 (31), 10568-10586. doi:10.1039/c9tc05415d

Hou, X., Zhang, Z., Wei, X., Qin, Y., Song, G., Li, L., et al. (2021). Aluminum Borate/ Boron Nitride Nanosheet Fibers for Enhancing the Thermal Conductivity of Polymer Composites. ACS Appl. Nano Mater. 4 (2), 2136-2142. doi:10.1021/acsanm.0c03429

Lee, D., Lee, B., Park, K. H., Ryu, H. J., Jeon, S., and Hong, S. H. (2015). Scalable Exfoliation Process for Highly Soluble boron Nitride Nanoplatelets by 
Hydroxide-Assisted ball Milling. Nano Lett. 15 (2), 1238-1244. doi:10.1021/ nl504397h

Li, T., Wang, L., Zhang, K., Xu, Y., Long, X., Gao, S., et al. (2016). Freestanding Boron Nitride Nanosheet Films for Ultrafast Oil/Water Separation. Small 12 (36), 4960-4965. doi:10.1002/smll.201601298

Li, Y., Zhu, H., Shen, F., Wan, J., Lacey, S., Fang, Z., et al. (2015). Nanocellulose as green Dispersant for Two-Dimensional Energy Materials. Nano Energy 13, 346-354. doi:10.1016/j.nanoen.2015.02.015

Morishita, T., and Okamoto, H. (2016). Facile Exfoliation and Noncovalent Superacid Functionalization of Boron Nitride Nanosheets and Their Use for Highly Thermally Conductive and Electrically Insulating Polymer Nanocomposites. ACS Appl. Mater. Inter. 8 (40), 27064-27073. doi:10.1021/ acsami.6b08404

Pan, D., Li, Q., Zhang, W., Dong, J., Su, F., Murugadoss, V., et al. (2021). Highly thermal Conductive Epoxy Nanocomposites Filled with 3D BN/C Spatial Network Prepared by Salt Template Assisted Method. Composites B: Eng. 209, 108609. doi:10.1016/j.compositesb.2021.108609

Shi, Y., Hamsen, C., Jia, X., Kim, K. K., Reina, A., Hofmann, M., et al. (2010). Synthesis of Few-Layer Hexagonal boron Nitride Thin Film by Chemical Vapor Deposition. Nano Lett. 10 (10), 4134-4139. doi:10.1021/nl1023707

Teng, C., Su, L., Chen, J., and Wang, J. (2019). Flexible, Thermally Conductive Layered Composite Films from Massively Exfoliated boron Nitride Nanosheets. Composites A: Appl. Sci. Manufacturing 124, 105498. doi:10.1016/ j.compositesa.2019.105498

Wang, M., Jiao, Z., Chen, Y., Hou, X., Fu, L., Wu, Y., et al. (2018). Enhanced thermal Conductivity of Poly(vinylidene Fluoride)/boron Nitride Nanosheet Composites at Low Filler Content. Composites Part A: Appl. Sci. Manufacturing 109, 321-329. doi:10.1016/j.compositesa.2018.03.023

Wu, K., Fang, J., Ma, J., Huang, R., Chai, S., Chen, F., et al. (2017). Achieving a Collapsible, Strong, and Highly Thermally Conductive Film Based on Oriented Functionalized Boron Nitride Nanosheets and Cellulose Nanofiber. ACS Appl. Mater. Inter. 9 (35), 30035-30045. doi:10.1021/ acsami.7b08214

Wu, K., Liao, P., Du, R., Zhang, Q., Chen, F., and Fu, Q. (2018). Preparation of a Thermally Conductive Biodegradable Cellulose Nanofiber/hydroxylated boron Nitride Nanosheet Film: the Critical Role of Edge-Hydroxylation. J. Mater. Chem. A. 6 (25), 11863-11873. doi:10.1039/c8ta03642j

Yan, Q., Alam, F. E., Gao, J., Dai, W., Tan, X., Lv, L., et al. (2021a). Soft and SelfAdhesive Thermal Interface Materials Based on Vertically Aligned, Covalently Bonded Graphene Nanowalls for Efficient Microelectronic Cooling. Adv. Funct. Mater. 31 (36), 2104062. doi:10.1002/adfm.202104062

Yan, Q., Dai, W., Gao, J., Tan, X., Lv, L., Ying, J., et al. (2021b). Ultrahigh-AspectRatio Boron Nitride Nanosheets Leading to Superhigh In-Plane Thermal Conductivity of Foldable Heat Spreader. ACS Nano 15 (4), 6489-6498. doi:10.1021/acsnano.0c09229
Yang, G., Zhang, X., Pan, D., Zhang, W., Shang, Y., Su, F., et al. (2021a). Highly Thermal Conductive Poly(vinyl Alcohol) Composites with Oriented Hybrid Networks: Silver Nanowire Bridged Boron Nitride Nanoplatelets. ACS Appl. Mater. Inter. 13 (27), 32286-32294. doi:10.1021/acsami.1c08408

Yang, G., Zhang, X., Shang, Y., Xu, P., Pan, D., Su, F., et al. (2021b). Highly Thermally Conductive Polyvinyl Alcohol/boron Nitride Nanocomposites with Interconnection Oriented boron Nitride Nanoplatelets. Composites Sci. Tech. 201, 108521. doi:10.1016/j.compscitech.2020.108521

Zeng, Z., Sun, T., Zhu, J., Huang, X., Yin, Z., Lu, G., et al. (2012). An Effective Method for the Fabrication of Few-Layer-Thick Inorganic Nanosheets. Angew. Chem. Int. Ed. 51 (36), 9052-9056. doi:10.1002/anie.201204208

Zeng, Z., Yin, Z., Huang, X., Li, H., He, Q., Lu, G., et al. (2011). Single-layer Semiconducting Nanosheets: High-Yield Preparation and Device Fabrication. Angew. Chem. Int. Ed. 50 (47), 11093-11097. doi:10.1002/anie.201106004

Zhang, X., Dong, J., Pan, D., Yang, G., Su, F., Ji, Y., et al. (2021). Constructing Dual thermal Conductive Networks in Electrospun Polyimide Membranes with Highly Thermally Conductivity but Electrical Insulation Properties. Adv. Compos. Hybrid Mater. 4 (4), 1102-1112. doi:10.1007/s42114-021-00335-9

Zhou, L., Yang, Z., Luo, W., Han, X., Jang, S.-H., Dai, J., et al. (2016). Thermally Conductive, Electrical Insulating, Optically Transparent Bi-layer Nanopaper. ACS Appl. Mater. Inter. 8 (42), 28838-28843. doi:10.1021/acsami.6b09471

Zhu, H., Li, Y., Fang, Z., Xu, J., Cao, F., Wan, J., et al. (2014). Highly Thermally Conductive Papers with Percolative Layered Boron Nitride Nanosheets. ACS Nano 8, 3606-3613. doi:10.1021/nn500134m

Zhu, W., Gao, X., Li, Q., Li, H., Chao, Y., Li, M., et al. (2016). Controlled Gas Exfoliation of Boron Nitride into Few-Layered Nanosheets. Angew. Chem. Int. Ed. 55 (36), 10766-10770. doi:10.1002/anie.201605515

Conflict of Interest: The authors declare that the research was conducted in the absence of any commercial or financial relationships that could be construed as a potential conflict of interest.

Publisher's Note: All claims expressed in this article are solely those of the authors and do not necessarily represent those of their affiliated organizations, or those of the publisher, the editors and the reviewers. Any product that may be evaluated in this article, or claim that may be made by its manufacturer, is not guaranteed or endorsed by the publisher.

Copyright (c) 2022 Kong, Li, Li, Xia, Wang, Wei, Xiong, Gong, Pan, Wu, Cai, Nishimura, Lin, Jiang and $Y u$. This is an open-access article distributed under the terms of the Creative Commons Attribution License (CC BY). The use, distribution or reproduction in other forums is permitted, provided the original author $(s)$ and the copyright owner(s) are credited and that the original publication in this journal is cited, in accordance with accepted academic practice. No use, distribution or reproduction is permitted which does not comply with these terms. 